\title{
Una lectura de las escuelas de formación deportiva y su contexto regulativo
}

\author{
A reading about Escuelas de Formación Deportiva and the regulation
}

\author{
Jairo Rosas Tibabuzo \\ Óscar H. Bernal Triviño \\ Efrain Serna Caldas
}

\section{Resumen}

En el presente artículo se pretende abrir un espacio de reflexión y diálogo sobre las Escuelas de Formación Deportiva (EFD), en particular con aquellos entes gubernamentales que dirigen, orientan y promueven las instituciones, organizaciones, asociaciones o movimientos que se han constituido con el propósito de formar a los niños y jóvenes de la capital; formación que por lo demás, se expresa contradictoria en relación con la orientación y la intención última de la misma. Tenemos así un sentido manifiesto por asegurar convicciones, modos de ser y hacer que coadyuven en la formación de ciudadanos y aseguren su participación integral en la sociedad, finalidad claramente educativa. El otro sentido expresa el interés definido de vincular a niños, niñas y jóvenes a aprendizajes deportivos y de esta manera, iniciar un proceso sistemático en el ámbito del deporte de rendimiento; se constituye asi una finalidad la formación de técnico laboral en el deporte. Una u otra finalidad implican procesos de saber y conocimiento comunes (constitución del sujeto, codeterminantes sociales y culturales) y a su vez diferenciales (procesos, valoraciones, metas, expectativas, modos de inserción social). Igualmente, un ejercicio profesional idóneo y competente para el alcance de sus fines.

Este primer momento de reflexión plantea el tema de los marcos de regulación a que se encuentran sujetas las políticas, los planes y programas de implementación del deporte, en particular, las disposiciones y normas que le dan vigencia, regulación y control a las Escuelas de Formación Deportiva (EFD) en nuestro país.

Palabras clave: Escuelas de formación deportiva, interpretación normativa.

\section{Abstract}

Key words: $\quad$ Sports formation schools, normative interpretation.

Fecha de recepción: 28 de octubre de 2005.

Fecha de aceptación: 8 de noviembre de 2005.

El equipo de investigación está conformado por los licenciados Oscar H. Bernal Triviño (investigador principal), Jairo Rosas Tibabuzo (coinvestigador), Efrain Serna Caldas (asesor), Andrés Castellanos y Juan Carlos Sarria y los monitores Schneider Contreras Leal, Francisco López Fierro estudiantes de Licenciatura de Educación Física, y Adriana Aguilar Medina, estudiante de noveno semestre de derecho, Universidad Católica. 


\section{Introducción}

El fenómeno deportivo ha invadido grandes esferas de la vida social (económica, política, social, cultural y educativa) de nuestro país y ha comprometido al Estado en su organización y control. Para ello, crea en 1968 el Instituto Colombiano para la Juventud y el Deporte (Coldeportes) como ente autónomo, adscrito al Ministerio de Educación Nacional (MEN). Entre las formas consolidadas de organización, sentidos y finalidades de orientación de las prácticas deportivas, en las décadas recientes, desarrolladas por dicho ente rector del deporte, se destaca el deporte formativo, cuyo propósito expreso consiste en hacer de las diferentes disciplinas deportivas un medio para el desarrollo integral, teniendo como foco de intervención principalmente niños, niñas y jóvenes de 9 a 14 años; para ello ha establecido lazos de organización con la institución escolar y ha introducido contenidos programáticos. Seguidamente se halla el deporte social o comunitario, en su pretensión de hacer de las actividades deportivas: medio de integración social, participación comunitaria, esparcimiento y recreo de comunidades locales y barriales. Como foco principal de intervención se encuentran los jóvenes y adultos, y sus principales formas de organización son agrupaciones o colectividades comunales, empresariales y șociales. Por último, el deporte competitivo, con su meta de formar deportistas de rendimiento y representación, ejercido principalmente por jóvenes y adultos en condiciones de nivel técnico calificado y el cual se organiza alrededor de clubes y ligas.

Entre estos sentidos y finalidades reclaman nuestro análisis inicial el deporte formativo, eje central de los procesos educativos, ejercido en particular en las escuelas de formación deportiva (EFD). En primera instancia, encontramos una proliferación de instituciones, organizaciones y asociaciones denotadas de finalidad formativa; una regulación débil para su creación, ejercicio, control y evaluación de su función educativa; ambigüedad entre su objeto misional expreso y su orientación y desarrollo real, y la ausencia de mecanismos sociales y de presencia de la sociedad civil, que aseguren su control y el cumplimiento de su finalidad.

En los primeros análisis y observaciones hechos a las EFD en relación con su finalidad, no hay definición clara de estas y sí, por el contrario se tiende a un eclecticismo de las mismas; es decir, se evidencian prácticas competitivas en asocio con ligas y federaciones, ejercicios de vínculos de promoción laboral a través de un sistema velado de promesas, $y$ la generación de expectativas de éxito temprano en el deporte, constituyendo un mundo ilusorio y de posibilidades irresolutas en niños, niñas y jóvenes.

Entablar una reflexión sobre las EFD es necesaria y pertinente a la misión educativa de la Universidad Pedagógica, y una responsabilidad social de las instituciones que se precian de tal misión, como de los entes que han delegado tan importante condición.

Un análisis a las escuelas de formación deportiva no puede abstraerse de los interrogantes bastante soslayados o presumiblemente resueltos: ¿Qué es una escuela de formación deportiva? ¿Cuáles son su origen y su naturaleza? ¿Qué tipo de formación se imparte en las EFD? ¿Qué papel desempeñan las EFD en la formación de niños, niñas y jóvenes las EFD?. Y si las EFD tienen como función primaria la formación de jóvenes y niños, sin duda, en ellas debe mediar una intención pedagógica que oriente sus procesos: ¿Qué concepción de desarrollo humano propician las EFD? ¿Qué capacidades potencian en los sujetos? ¿Cuáles son los procesos de evaluación de los propósitos y las metas de las EFD? ¿Qué garantías sociales se tienen con respecto a las $\mathrm{EFD}$ ?. Éstos y otros interrogantes generales son los que conforman el centro de interés de esta investigación.

La definición, la importancia y la expansión de las EFD es comprensible, inicialmente en el marco de relevancia que el deporte posee actualmente. La práctica de los deportes ha desbordado los límites de las fronteras de países y se ha instalado de manera sutil pero muy profunda, en lo más profundo de la realidad social, haciendo parte de las prácticas cotidianas que movilizan las expectativas, los intereses e incluso las necesidades de todas las personas.

\section{Universalización de la práctica deportiva}

Los deportes se han incrustado en la vida diaria de personas y sociedades derivando en instituciones y manifestaciones, que van desde las prácticas del deporte de competencia que llena estadios como una de las principales formas de consumo de espectáculo y maximización el rendimiento corporal, pasando por clubes y los torneos de fin de semana de practicantes-deportistas emulando y representando al mundo del deportista de rendimiento, hasta llegar a la escuela, donde ha desplazado el juego libre, para convertirse en estructura programática de la clase de educación física.

Su universalización es cada vez más ostensible como producto del desarrollo de la organización deportiva 
Comité Olímpico Internacional (COI) y de la sensibilización de instituciones que como la ONU confiere al deporte. La Carta Internacional de la Educación Física y el Deporte (Unesco, 1978) así lo ratifica en su artículo primero:

"La Educación Física y la práctica de los deportes son un derecho fundamental de todo el mundo".

$\mathrm{Al}$ definirse como un derecho fundamental de todo el mundo, promociona y eleva la práctica de los deportes a la condición de un derecho inherente a la persona humana, por tanto, necesario, para que ella cuente con una vida digna.

Si bien tales orientaciones no obligan a los países, sí los comprometen en su condición de miembros de la Organización de las Naciones Unidas (ONU), en particular en sus propósitos de mantener la paz y la seguridad internacionales, desarrollar relaciones de amistad entre las naciones, alcanzar una cooperación internacional fundada sobre las relaciones de amistad entre las naciones, alcanzar una cooperación internacional en la solución de problemas económicos, sociales, culturales o humanitarios y fomentar el respeto por los derechos humanos y las libertades fundamentales.

Como directrices, los diferentes estados asumen estas recomendaciones, las implementan según condiciones $\mathrm{y}$ posibilidades internas $\mathrm{y}$ derivan planes que se traducen en proyectos concretos de promoción y práctica del deporte como lo sugiere el mencionado organismo.

Entre las actuales orientaciones figuran como producto del informe: "Deporte para el Desarrollo y la Paz". Hacia el cumplimiento de los objetivos de Desarrollo del Milenio, se concluye "que el deporte - desde el juego y la actividad física hasta el deporte organizado y competitivo- es una herramienta poderosa y rentable para avanzar hacia los objetivos de Desarrollo para el Milenio" (Revista Unicef: 2004). Además, el secretario general de las Naciones Unidas elaboró unas recomendaciones sobre el deporte para el desarrollo y la paz, que se registran en seis puntos como directriz de lo que podría ser una política internacional para la implementación y la promoción del deporte en los países del mundo. Ellos son:

1. Incorporar el deporte y la actividad física en el programa de desarrollo de los países y los organismos nacionales e internacionales de desarrollo, haciendo un énfasis particular en la juventud.

2. Convertir la práctica del deporte en un propósito y en una herramienta para lograr los objetivos de Desarrollo para el Milenio y las metas de otras conferencias internacionales y alcanzar las metas más amplias de desarrollo y paz.
3. Incluir las iniciativas relacionadas con el deporte en los programas de los organismos de las Naciones Unidas siempre que sea apropiado, y de acuerdo con la evaluación de las necesidades locales.

4. Facilitar el establecimiento de alianzas innovadoras que incluyan el deporte como herramienta de desarrollo.

5. Como parte de la asistencia para el desarrollo en el exterior, instar a los gobiernos a destinar recursos a iniciativas y programas deportivos para el desarrollo que traten de lograr la máxima participación y acceso al "deporte para todos" en sus propios países, y fortalecer la posición del deporte y la actividad física en la formulación general de las políticas. Así mismo, ungir a los aliados del sistema de la Naciones Unidas incluidos el sector privado, las organizaciones deportivas y la sociedad civil a dar apoyo financiero y en especial para el deporte a favor del desarrollo y la paz.

6. Alentar al sistema de las Naciones Unidas a buscar mecanismos novedosos para hacer del deporte un medio de comunicación y movilización social en los ámbitos nacional, regional y local.

\section{La práctica deportiva, un derecho en Colombia}

La Constitución política de Colombia (CPC) definió al Estado colombiano como Estado social de derecho, con ello pretendió fundir dos grandes aspiraciones:

De un lado, la búsqueda de la igualdad, por eso se dice social; $y$, de otro la garantía de la libertad bajo el imperio de las leyes, por lo cual se llama de derecho. (Castellanos: 1994, 52).

Un Estado que al tiempo que defiende y promulga los derechos civiles y políticos individuales, se compromete en la realización de los derechos económicos y sociales. Por consiguiente, se propone garantizar el bienestar de la población e intervenir en el curso de la sociedad, y las políticas sociales, de seguridad, empleo educación, etc., son su preocupación central. De esta manera, el Estado social de derecho considera a la persona humana en sus diversas manifestaciones, individuales y colectivas, y el reconocimiento de sus derechos en ambas esferas. $\mathrm{Al}$ considerarse el deporte como una de las manifestaciones del ser humano en su relación consigo mismo y con los demás puede interpretarse entonces que le asiste a los ciudadanos colombianos, el derecho de todas las personas, a la práctica del deporte.

Con la Constitución política de Colombia de 1991, se dio el primer paso para constituir la práctica del depor- 
te como derecho social, económico y cultural, promulgando su artículo 52, que reza:

Se reconoce el derecho de todas las personas a la recreación, la práctica del deporte y el aprovechamiento del tiempo libre. El Estado fomentará estas actividades e inspeccionará las organizaciones deportivas, cuya estructura y propiedad deberán ser democráticas.

Otro aspecto para destacar del mencionado artículo es lo referido a la estructura y la propiedad de las organizaciones deportivas, donde enfatizan su carácter democrático; en esencia, se interpreta el deseo por parte de los constituyentes de preservar y mantener el ideal de lo social de estas organizaciones; se estimula la asociación de los particulares y les garantiza espacios y formas de representación para que incidan en el curso de las decisiones sociales.

Debido a la complejidad manifiesta de la práctica deportiva y su importancia capital para la persona, dicho derecho, a pesar de haber sido incluido en el título II, capítulo 2, "De los derechos sociales, económicos y culturales", puede ser contemplado como un derecho fundamental por conexidad con los mismos, en la medida en que la opción del sujeto por una práctica deportiva encuentra amparo en el derecho al libre desarrollo de la personalidad; por el derecho a la libre asociación que encuentra su asidero en las organizaciones deportivas creadas por los particulares con el objeto de promover o regular la práctica social e individual del deporte.

Además, la práctica deportiva significa para muchas personas el medio del propio sustento vital y la forma de acceder al trabajo; por su valor formativo este derecho hace parte de educación, y constituye así un elemento fundamental del sistema educativo. Su práctica es importante en el mantenimiento de la salud, por tanto, es un factor corrector de desequilibrios sociales que contribuye al desarrollo de la igualdad entre los ciudadanos, crea hábitos favorecedores de la inserción social, y su práctica colectiva fomenta la solidaridad.

\section{La práctica deportiva, una función expresa de desarrollo y formación}

Son muy frecuentes las discusiones y los debates en torno del sentido de las prácticas deportivas, en particular las de las EFD. Como punto de partida, ellas deben comprometerse con la función educativa y formativa de niños, niñas y jóvenes. Este punto de vista se hace expreso a partir de la reforma del artículo 52 de la Constitución política de Colombia, mediante el acto legislativo
No. 02 de agosto 17 de 2000, y queda así:

Artículo 52. El ejercicio del deporte, sus manifestaciones recreativas, competitivas y autóctonas tienen como función la formación integral de las personas, preservar y desarrollar una mejor salud en el ser humano. El deporte y la recreación forman parte de la educación y constituyen gasto público social.

Se reconoce el derecho de todas las personas a la recreación, a la práctica del deporte y al aprovechamiento del tiempo libre. El Estado fomentará estas actividades e inspeccionará y vigilará las organizaciones deportivas y recreativas, cuya estructura y propiedad deberán ser democráticas.

\section{¿Qué implicaciones o consideraciones pueden reali- zarse a partir de esta modificación?}

Esta modificación del artículo implica una precisión frente a la función de las prácticas deportivas de la contemplada inicialmente reconociendo la importancia de este derecho en la formación de la persona. Al integrar el deporte a la educación, establece una directriz clara y un deseo expreso de propiciar en su aplicación la formación de un sujeto coherente con el ideal educativo que se explicita en la constitución misma y que, por consiguiente, toda la estructura deportiva del país debe armonizarse con las diferentes modalidades educativas.

En el artículo 67 de la CPC se definen las principales finalidades educativas y se consagra el derecho a la educación y que el inciso 2 establece:

La educación formará al colombiano en el respeto a los derechos humanos, a la paz y a la democracia; y en la práctica del trabajo y la recreación, para el mejoramiento cultural, científico, tecnológico y para la protección del ambiente.

Se deja plasmado en este artículo el ideal de ciudadano colombiano y los aspectos más relevantes de su formación, la cual debe ser contemplada y asumida por todas las instituciones y organizaciones que intervengan en su proceso. Al ser así, establece una condición necesaria que debe asumir toda institución, persona o entidad que se promueva como formadora de los sujetos en nuestra sociedad.

Así mismo, en correspondencia a la constitución, la Ley 115 de febrero 8 de 1994, por la cual se expide la Ley General de Educación, establece unos principios orientadores que de una manera concreta redefinen la estructura del sistema educativo, sus fines, los niveles y los ciclos y las modalidades educativas. Su artículo 5 los prescribe así: 
1. El pleno desarrollo de la personalidad sin más limitaciones que las que le imponen los derechos de los demás y el orden jurídico, dentro de un proceso de formación integral, física, psíquica, intelectual, moral, espiritual, social, afectiva, ética, cívica y demás valores humanos.

2. La formación en el respeto a la vida y a los demás derechos humanos, a la paz, a los principios democráticos, de convivencia, pluralismo, justicia, solidaridad y equidad, así como en el ejercicio de la tolerancia y de la libertad.

3. La formación para facilitar la participación de todos en las decisiones que los afectan en la vida económica, política, administrativa y cultural de la Nación.

4. La formación en el respeto a la autoridad legítima y a la ley, a la cultura nacional, a la historia colombiana y a los símbolos patrios.

5. La adquisición y la generación de los conocimientos científicos y técnicos más avanzados, humanisticos, históricos, sociales, geográficos y estéticos, mediante la apropiación de hábitos intelectuales adecuados para el desarrollo del saber.

6. El estudio y la comprensión crítica de la cultura nacional y de la diversidad étnica y cultural del país, como fundamento de la unidad nacional y de su identidad.

7. El acceso al conocimiento, la ciencia, la técnica y demás bienes y valores de la cultura, el fomento e la investigación y el estímulo a la creación artística en sus diferentes manifestaciones.

8. La creación y fomento de una conciencia de la soberanía nacional y para la práctica de la solidaridad y la integración con el mundo, en especial con Latinoamérica y el Caribe.

9. El desarrollo de la capacidad crítica, reflexiva y analítica que fortalezca el avance científico y tecnológico nacional, orientado con prioridad al mejoramiento cultural y de la calidad de vida de la población, a la participación en la búsqueda de alternativas de solución a los problemas y al progreso social y económico del país.

10. La adquisición de una conciencia para la conservación, protección y mejoramiento del medio ambiente, de la calidad de vida, del uso racional de los recursos naturales, de la prevención de desastres, dentro de una cultura ecológica y del riesgo y la defensa del patrimonio cultural de la Nación.

11. La formación en la práctica del trabajo, mediante los conocimientos técnicos y las habilidades, así como en la valoración del mismo como fundamento del desarrollo individual y social.

12. La formación para la promoción y preservación de la salud y la higiene, la prevención integral de pro- blemas socialmente relevantes, la educación física, la recreación, el deporte y la utilización adecuada del tiempo libre, $y$

13. La promoción en la persona y en la sociedad de la capacidad para crear, investigar, adoptar la tecnología que se requiere en los procesos de desarrollo del pais y que le permita al educando ingresar al sector productivo.

Como consecuencia, al tener las prácticas deportivas expresas sus finalidades de formación integral, necesariamente deben articularse a las consideraciones de sus finalidades, y de esta forma, al ser las EFD expresiones concretas de práctica deportiva, obliga la consecución de tales finalidades.

Finalmente, en el artículo 52 se obliga al Estado en su responsabilidad concreta del ejercicio del derecho al ejercicio del deporte, constituyéndolo en gasto público social y campo objeto de control y regulación. Que sea gasto público social implica que esté contemplado en el presupuesto nacional, que destine mediante el Estatuto Tributario, un porcentaje de los ingresos anuales de la nación; y tenga un propósito de utilidad pública, para la formulación de sus políticas, el fortalecimiento de sus instituciones y la garantía del acceso de todas las personas al ejercicio real de este derecho.

\section{El deporte formativo}

La Ley 181 de 1995 se expidió con un objetivo específico, crear el Sistema Nacional del Deporte; y dos objetivos generales: de un lado, el patrocinio, el fomento, la masificación, la divulgación, la planificación, la coordinación, la ejecución y el asesoramiento de la práctica del deporte, la recreación y el aprovechamiento del tiempo libre, y, del otro lado la promoción de la educación extraescolar de la niñez y la juventud en todos los niveles y estamentos sociales del país.

En ella se define la educación extraescolar como la educación que utiliza el tiempo libre, la recreación y el deporte como instrumentos fundamentales para la formación integral de la niñez y de los jóvenes y para la transformación del mundo juvenil con el propósito de que éste incorpore sus ideas, sus valores y su propio dinamismo interno al proceso de desarrollo de la nación. Esta educación complementa las que dan la familia y la escuela y se realiza por medio de organizaciones, asociaciones o movimientos para la niñez o de la juventud e instituciones sin ánimo de lucro que tengan como objetivo prestar este servicio a las nuevas generaciones. 
A pesar de que la educación extraescolar no es referida en la Ley General de Educación, se retoma en la ley 375 de 1997 o Ley de la Juventud, considerándola como la acción pedagógica sin carácter obligatorio, de libre adhesión y durante el tiempo libre, que busca la formación integral de los jóvenes y la transformación del mundo juvenil en fuerza educativa al servicio del desarrollo del país. Esta ley adiciona la educación extraescolar como una modalidad de la formación integral independiente a las modalidades de educación formal, no formal e informal que establece la Ley General de Educación como se desprende de su artículo 37:

La formación integral de la juventud debe desarrollarse en las modalidades de educación extraescolar, y en las modalidades de educación formal, no formal e informal.

La educación extraescolar, aunque no esté reglamentada y pueda considerarse distinta de las modalidades comprendidas por la Ley General de Educación, necesariamente deben cumplir con el objeto y los fines de la educación, como son: el pleno desarrollo de la personalidad sin más limitaciones que las que le imponen los derechos de los demás y el orden jurídico, en un proceso de formación integral, la formación en el respeto a la vida y a los demás derechos humanos, la formación en el respeto a la autoridad legítima y a la ley, a la cultura na= cional, a la historia colombiana y a los símbolos patrios, el estudio y la comprensión crítica de la cultura nacional y de la diversidad étnica y cultural del país, la adquisición de una conciencia para la conservación, protección y mejoramiento del medio ambiente, la formación en la práctica del trabajo, mediante los conocimientos técnicos y habilidades, la formación para la promoción y preservación de la salud y la higiene, la prevención integral de problemas socialmente relevantes, la educación física, la recreación, el deporte y la utilización adecuada del tiempo libre, etc. (Ley 115: 1994, arts. 1 y 5). Por tanto, existe la necesidad de su regulación como modalidad de la educación y su vigilancia como tal, de tal manera que se incorporen en su control y vigilancia entidades oficiales que garanticen el cumplimiento de sus objetivos.

La legislación establece diversas formas en las cuales se desarrolla el deporte (Ley 181: 1995, art. 16), entre ellas el deporte formativo, el cual define como "aquel que tiene como finalidad contribuir al desarrollo integral del individuo. Comprende los procesos de iniciación, fundamentación y perfeccionamiento deportivos. Tiene lugar tanto en los programas del sector educativo formal y no formal, como en los programas desescola- rizados de las escuelas de formación deportiva y semejantes".

\section{El deporte formativo desde la norma}

El deporte formativo hace parte del Sistema Nacional del Deporte y planifica, en concordancia con el Ministerio de Educación Nacional (MEN), la enseñanza y la utilización constructiva del tiempo libre y la educación en el ambiente, para el perfeccionamiento personal y el servicio a la comunidad. El Instituto Colombiano del Deporte (Coldeportes), es el máximo organismo planificador, rector, director y coordinador del Sistema Nacional del Deporte y director del deporte formativo.

El objetivo fundamental de la ley 181 fue crear el Sistema Nacional del Deporte, que es el conjunto de organismos, articulados entre sí, para permitir el acceso de la comunidad al deporte, la recreación, el aprovechamiento del tiempo libre, la educación extraescolar y la educación física. Este sistema tiene como objetivo generar y dar a la comunidad oportunidades de participación en procesos de iniciación, formación, fomento y práctica del deporte. Lo conforman el Ministerio de Educación Nacional, el Instituto Colombiano del Deporte (Coldeportes), los entes departamentales, municipales y distritales que ejerzan las funciones de fomento, desarrollo y práctica del deporte, la recreación y el aprovechamiento del tiempo libre, los organismos privados, las entidades mixtas, así como todas las entidades públicas o privadas de otros sectores sociales y económicos en los aspectos que se relacionen directamente con estas actividades.

\section{Escuelas de formación deportiva}

El decreto 2225 de 1985 se promulgó con el fin de reglamentar parcialmente el decreto 2845 de 1984 y por el cual se dictan disposiciones sobre la participación de niños y niñas en eventos deportivos. Este ha servido de referente legal para que el Instituto Distrital para la Recreación y el Deporte asuma la dirección, la regulación y el control de las escuelas de formación deportiva. Según este decreto, el programa de Formación deportiva de las escuelas de formación es de carácter extracurricular y complementa la formación física y deportiva, su objetivo es desarrollar procesos pedagógicos, técnicos, deportivos, sociales, culturales y morales en los niños, niñas y jóvenes mediante de acciones formativas de movimiento, garantizando el mejoramiento de la calidad de vida y la adquisición de fundamentos técnicos y tácticos en el deporte (Decreto 2225: 1985). 
La formación comprende los procesos de irradiación, iniciación, fundamentación y perfeccionamiento deportivos. La irradiación es el proceso pedagógico deportivo mediante el cuál se efectúa una extensa estimulación de las habilidades motrices deportivas, evitando los estereotipos propios de una sola modalidad deportiva. El proceso de iniciación tiene por objeto el desarrollo sicomotor de niños, niñas y jóvenes, complementar la educación física y los hábitos de práctica deportiva, fomentar hábitos deportivos, el trabajo en equipo, la capacidad de superación, el desarrollo de habilidades y destrezas, el mejoramiento de la salud y la formación deportiva y humana, no exclusivamente competitiva. En el proceso de fundamentación se proporcionan las bases para la fundamentación de las técnicas características un deporte específico. Con el proceso de perfeccionamiento deportivo se afianzan los elementos aprendidos y vivenciados, su objetivo es identificar futuros deportistas para perfeccionarlos y tener la posibilidad de alcanzar un alto nivel deportivo.

Partiendo de la determinación de estos procesos por parte de la legislación y de la libertad que confiere la ley a Coldeportes para fijar las edades y ajustar los procesos, el IDRD estableció los niveles de aprendizaje. Estos niveles son: nivel de iniciación deportiva, nivel de especialización deportiva y nivel de perfeccionamiento deportivo.

En el nivel de iniciación deportiva se estimulan las habilidades motrices evitando los estereotipos de una sola modalidad deportiva, para lograr las bases de la formación motriz. El proyecto pedagógico de este nivel debe potenciar las dimensiones comunicativa, cognitiva, ética, estética, corporal y lúdica; las edades de práctica en este nivel oscilan entre los 6 y los 12 años. Este nivel comprende los procesos de irradiación e iniciación de los que habla la ley.

En el nivel de especialización deportiva se adquiere la maduración y el dominio de los aspectos motores básicos y deportivos y se incorporan mayores exigencias físicas, técnicas y tácticas en el deporte elegido. Por tanto, la especialización es el nivel donde se consolida la fundamentación técnico-táctica en el deporte. Las edades de este nivel oscilan entre los 12 y los 18 años. En este nivel se incluye el proceso de fundamentación, inclinándolo más hacia la especialización deportiva y la competencia. El nivel de perfeccionamiento deportivo está dirigido a una población previamente seleccionada para un deporte desde los 12 años en adelante. Los participantes ingresan, previa valoración de su aptitudes funcional, psíquica y cognitiva determinadas según la modalidad deportiva; esto implica en la práctica procesos de alto rendimiento con cargas de entrenamiento mínimo de entre dos y tres horas diarias, con una frecuencia establecida en cada escuela de acuerdo con la preparación física establecida por el profesional encargado del entrenamiento, y exige la participación de los deportistas en eventos nacionales e internacionales del ciclo olímpico. La edad coincide en este nivel con la edad del nivel de especialización, debido a que en este nivel participan los deportistas escogidos por sus habilidades entre los niños y niñas pertenecientes al nivel de especialización.

El IDRD ha determinado en estos niveles con el fin de unificar criterios sobre la estructura organizativa con el fin de dar un soporte o directriz para las escuelas de formación deportiva, el análisis correspondiente a dicha estructura es la priorización de contenidos centrados en la adquisición de destrezas técnico-deportivas, omitiendo la argumentación inicial sobre la formación integral de los niños y niñas participantes $\mathrm{y}$, por tanto, deja de lado procesos pedagógicos, sociales, culturales y éticos.

La ley más reciente emitida por el Congreso de Colombia, la Ley 934 (2004), la cual se encuentra en proceso de implementación, retoma algunos lineamientos para las escuelas de formación deportiva en los siguientes artículos:

Artículo 2. Todo establecimiento - educativo del pais deberá incluir en su proyecto educativo institucional, PEI, además del plan integral del área de la educación física, recreación y deporte, las acciones o proyectos pedagógicos complementarios del área. Dichos proyectos se desarrollarán en todos los niveles educativos con que cuenta la institución y propenderá a la integración de la comunidad educativa.

Artículo 5. Para propender al desarrollo de la Educación Física en la comunidad, partiendo de la base de la población infantil escolar como extraescolar, se adoptarán y fortalecerán los centros de educación física que articulen sus servicios con los programas establecidos en el proyecto educativo institucional, PEI, de los establecimientos educativos. En igual forma se procederá, respecto de los Centros de Iniciación y Formación Deportiva, adscritos a los entes deportivos municipales.

Parágrafo 1. El Programa de Centros de Educación Física es una estrategia pedagógico-metodológica, donde participan entidades e instituciones educativas de las zonas urbanas y rurales de cada municipio, que en una y otra forma intervienen en el desarrollo curricular y pedagógico de la educación 
física, la recreación y el deporte, aplicando criterios técnicos, científicos, tecnológicos y lúdicos, contribuyendo así al desarrollo técnico integral.

Parágrafo 2. El Programa de Centros de Iniciación y Formación Deportiva es de carácter formativo extracurricular y complementa la formación física y deportiva de la población infantil, contribuyendo a su desarrollo motor, en las distintas etapas de crecimiento (iniciación y formación).

En la presente ley se distingue la intención de diferenciar los ámbitos en que el deporte puede hacer presencia en los procesos de desarrollo de los niños, niñas y jóvenes (artículo 2), en primera instancia se hace alusión especial a integrar en los proyectos educativos institucionales de los colegios y escuelas los programas y proyectos que atiendan la formación integral, por tanto, deben estar inscritos en la orientación pedagógica de la institución educativa y sujetos a las disposiciones de la institución. Con este enfoque del deporte en la escuela se afianza la idea expuesta en el texto sobre los Lineamientos curriculares para la educación física. Recreación y deportes (Lineamientos curriculares: 2000, 87).

Las escuelas de formación deportiva constituyen en una opción para la práctica de los deportes con un carácter formativo que requieren la integración de la comunidad y la coordinación interinstitucional para darle solidez y permanencia, fundamentadas en los mismos lineamientos curriculares establecidos para la educación física en su conjunto.

En segunda instancia se hace alusión a la formación deportiva o formación para el deporte, donde se prioriza la adquisición de ciertas destrezas técnico-deportivas en busca de un rendimiento que le permita la participante escalar en las estructuras del deporte federado, por tanto, delega dicho proceso a los clubes deportivos cuya finalidad sería la promoción de los deportistas para responder a la estructura del deporte de rendimiento y cuyo énfasis estaría centrado en la competencia.

\section{Personería jurídica de las escuelas de formación deportiva}

Los clubes deportivos y promotores del orden municipal y las escuelas de formación deportiva sólo están obligados a obtener personerías jurídicas y organizarse como corporaciones deportivas para acceder a recursos públicos y en los demás eventos que expresamente la ley determine, como en el caso de las donaciones, es decir, que no es requisito obtener la personería si la escuela o club (municipal) no lo desea.
La ley 24 de 1988, en su artículo 55, determina: “Corresponde otorgar las personerías jurídicas de las fundaciones de recreación y deporte de nivel municipal y al Gobernador del respectivo departamento otorgársela a las de nivel departamental".

Posteriormente, con la ley 181 de 1995, que creo el Sistema Nacional del Deporte, y en los decretos 1227, 1228 y 1229 de 1995, que desarrollaron dicha ley, se estableció que la personería jurídica de los organismos deportivos de carácter nacional será otorgada por el Instituto Colombiano del Deporte (Coldeportes), y la de los organismos deportivos de los demás órdenes, por las autoridades competentes del respectivo orden; por tanto, al constituir una legislación especial saca a las fundaciones de recreación y deporte del control y vigilancia de la cámaras de comercio y las Superintendencias y se la asigna al mismo Coldeportes. De modo que aunque no se ha derogado expresamente la Ley 24 del 1988 se aplica la Ley 181, que es posterior y especial.

\section{Inspección, vigilancia y control}

El Instituto Colombiano del Deporte (Coldeportes), por delegación del Presidente de la República, ejercerá las funciones de inspección, vigilancia y control de los organismos deportivos y demás entidades del Sistema Nacional del Deporte (Decreto 1227. 1995. arts. 1 y 2).

La inspección, la vigilancia y el control se ejercerán mediante el requerimiento de informes de sus actividades, solicitud de información jurídica, financiera, administrativa y contable relacionada con el objeto social y su desarrollo, visitas de inspección con el fin de comprobar el cumplimiento de las disposiciones legales y estatutarias.

Igualmente, puede realizar una intervención de las autoridades competentes para la cancelación de eventos deportivos de carácter nacional y, por último, la solicitud a las autoridades competentes de suspender de modo temporal a los órganos directivos, administradores y de control, cuando medie investigación disciplinaria o penal, y exista pliego de cargos o vinculación formal al respectivo proceso penal.

Conforme a la jurisprudencia, es claro que el Estado tiene la facultad de inspeccionar las instituciones deportivas y la actividad deportiva y que, en principio, esa función de inspección, vigilancia y control está radicada en cabeza del Presidente de la República, pues, conforme a la propia Ley del Deporte, tales clubes son corporaciones sin ánimo de lucro o sociedades anónimas, las cuáles están sujetas a vigilancia presidencial. 
Sin embargo, en la Constitución política de Colombia es claro que la delegación es factible, pues el artículo 211 autoriza la delegación presidencial también en los representantes legales de entidades descentralizadas.

Ahora bien, conforme a la ley 181 de 1995 , Coldeportes es un establecimiento público del orden nacional, por lo cual ninguna objeción constitucional puede hacerse a que la ley delegue en esa entidad la inspección, la vigilancia y el control sobre los organismos deportivos y demás entidades que conforman el Sistema Nacional del Deporte. Aunque se discute sobre la eficiencia y la conveniencia de que una misma institución regule, otorgue las personerías y controle y vigile sus propias actuaciones.

\section{Políticas del deporte}

El deporte y todo lo relacionado con él constituyen una función pública del Estado que ejerce el Ministerio de Educación por conducto de Coldeportes, éstos entes en coordinación deben fijar las políticas en materia de deporte y fomentar la educación extraescolar, por tanto, el deporte formativo y las otras formas de deporte social comunitario, universitario, asociado, competitivo, de alto rendimiento, aficionado, profesional, entre otros (Ley 181: 1995. art. 16), no pueden estar desligadas de los fines y propósitos educativos.

Las políticas del deporte en Colombia deben armonizarse con las políticas educativas, aun cuando Coldeportes, como entidad pública, tenga personería jurídica, autonomía administrativa y patrimonio independiente, es un establecimiento público del orden nacional adscrito al Ministerio de Educación Nacional, está sujeto a control de parte de los diferentes organismos de control del Estado, y la definición de sus planes, políticas y estrategias deben ser acordes con los lineamientos que la nación le indique por medio del Ministerio de Educación. La misma ley 181 de 1995 asigna al Ministerio de Educación Nacional competencias administrativas, así:

Articulo 59. Corresponde al Ministerio de Educación Nacional, en coordinación con Coldeportes:

1. Diseñar las políticas y metas en materia de deporte, recreación, el aprovechamiento del tiempo libre y la educación física para los niveles que conforman el sector educativo.

2. Fijar los criterios generales que permitan a los departamentos regular, en concordancia con los municipios y de acuerdo con esta ley, la actividad referente al deporte, la recreación, el aprovechamiento del tiempo libre y la educación física en el sector educativo.
¿Por qué el Ministerio delega o coordina con una entidad cuyo carácter no es educativo, como Coldeportes, diseñar las políticas y metas en materia de deporte, recreación, aprovechamiento del tiempo libre y la educación física? Esta es una inquietud que merece resaltarse y que se desprende del artículo anterior. Podría decirse que existe una contradicción en la delegación de funciones y la naturaleza misma de la institución; la cual se resuelve porque el MEN puede delegar funciones, $y$ en consideración a la necesidad para el buen desempeño y cumplimiento de dichas funciones, éstas deben asignarse a entidades que tengan como eje de acción la formación y la pedagogía misma en atención al deseo expreso de la carta constitucional de integrar dichas prácticas al proceso formativo de los sujetos.

Por otra parte, en la norma no es clara en determinar qué funciones y hasta donde va el límite de dicha delegación o coordinación. Lo que sí es cierto es la necesidad de clarificar el límite de cada organismo en cumplimiento de sus funciones y, por lo que podría interpretarse por la naturaleza de las mismas, es una división con relación a la orientación y la definición de políticas y la proposición de planes y programas que atiendan de manera particular la pertinencia de su función. Al MEN debería corresponder todo lo concerniente con la formación de sujetos en la sociedad, es decir, el deporte en el ámbito de lo formativo como una alternativa para concretar el ideal de ciudadano colombiano, y a Coldeportes el desarrollo y la promoción del deporte en el ámbito laboral.

\section{Consideraciones finales}

De eesta revisión de las normas y los artículos que dan el marco legal al deporte formativo y a las escuelas de formación deportiva, surgen algunos puntos de análisis que se ponen en consideración, con el propósito de realizar la respectiva reflexión desde el ámbito de la pedagogía.

1. Las políticas deportivas deben enmarcarse en un espectro amplio de las finalidades educativas y corresponder a los proyectos de nación y sociedad que enmarca el Estado Social de derecho. En procura de la construcción de una sociedad más tolerante, participativa y democrática, como se demanda en la Constitución, todos los espacios de formación que la sociedad acepta y promueve deben comprometerse con este propósito; por consiguiente, el deporte, desde su enfoque como factor de formación, debe que necesariamente comprometerse con ser facilitador 
de procesos que permitan la concreción de dicho propósito.

2. Si la escuela de formación deportiva obedece a un proyecto social educativo, debe estar enmarcada en un proyecto educativo institucional de una escuela, colegio, universidad o institución con reconocimiento por parte de las entidades gubernamentales, departamentales, distritales o locales encargadas de su reconocimiento y que avala su acción ante la comunidad.

Con base en lo anterior, se considera que su actividad también debe estar regulada y orientada por la institución educativa a la cual está inscrita; por consiguiente, debe estar en concordancia con la misión y la visión de la institución y propender al desarrollo de los jóvenes. Debe estar vinculada a un proyecto educativo para evitar la circunstancia de presentar inconsistencias entre la orientación del proyecto educativo de la institución, que de una manera u otra debe constituir el eje de la formación de la comunidad y las diversas visiones y prácticas que realizan las escuelas de formación deportiva.

3. Se percibe una incoherencia en la exposición de la definición del deporte formativo (ley 181 y decreto 2225), en los cuales se concibe el deporte formativo como proceso pedagógico que propende al desarro-

\section{Bibliografía}

CASA NACIONAL DEL PROFESOR (Canapro). (2003). Legislación educativa colombiana, 1991-2003. Bogotá. Editorial Canapro.

CASTELLANOS, Camilo. (1994). Instituto para el Desarrollo de la democracia Luis Carlos Galán Sarmiento. Proyecto escuela para la democracia y la convivencia.

INSTITUTO COLOMBIANO DEL DEPORTE (Coldeportes). (2001). Legislación deportiva ley 181 de 1995 decretos leyes y sus decretos reglamentarios. Armenia. Editorial Kinesis.

CHINCHILLA, Víctor, GÓMEZ, Laureano y SÁNCHEZ, Henry. (2000). Lineamientos curriculares. educación física, recreación y deportes. Ministerio de Educación Nacional. Bogotá,. Cooperativa Editorial del Magisterio.

\section{Legislación}

Acto Legislativo No. 02 DE 2002. Ministerio de Educación Nacional (2002).

Decreto reglamentario 1227 de 1995. Ministerio de Educación Nacional.

Decreto Ley 1228 de 1995. Ministerio de Educación Nacional.

Decreto reglamentario 00407 de 1996. Ministerio de Educación Nacional.

Ley 181 de 1995. Congreso de la República.

Ley 494 de 1999. Congreso de la República. llo integral de niños, niñas y jóvenes participantes, pero en los elementos que lo constituyen y los niveles de trabajo se argumentan exclusivamente procesos de adquisición de habilidades técnico-deportivas, sin dar cuenta de los procesos de formación del niño o niña en los aspectos sociales, cognitivos, afectivos, etc.; por tanto, se evidencia una notoria tendencia a la atención del rendimiento deportivo, lo cual confunde su esencia y su propósito como medio para la formación.

\section{Colaboradores}

Andrés Castellanos. Profesor catedrático de la Universidad Pedagógica Nacional. Bogotá.

Juan Carlos Sarria. Profesor catedrático de la Universidad Pedagógica Nacional. Bogotá.

Schneider Contreras Leal. Monitor de investigación y estudiante de octavo semestre de Licenciatura en Educación Física. Universidad Pedagógica Nacional. Bogotá.

Francisco López Fierro. Monitor de investigación y estudiante de octavo semestre de Licenciatura en Educación Física. Universidad Pedagógica Nacional. Bogotá. Adriana Aguilar Medina. Estudiante de noveno semestre de derecho, Universidad Católica. Bogotá.

MINISTERIO DE EDUCACIÓN NACIONAL. Chinchilla, Victor, Gómez, Laureano y Sánchez, Henry. (2000). Lineamientos curriculares. educación física, recreación y deportes.

MINISTERIO DE EDUCACIÓN NACIONAL. (2000). Lineamientos curriculares educación física, recreación y deportes. Bogotá. Cooperativa Editorial del Magisterio.

MUÑOZ, José. (2001). Definición de deporte (artículo). Revista digital EF Deportes.

REVISTA UNICEF. (2004). Fondo de las Naciones Unidas para la Infancia. División de Comunicaciones. Nueva York. pubdoc@ unicef.org.

Ley 375 de 1997. Congreso de la República.

Ley 934 de 2004. Congreso de la República.

Ley 115 de 1994, artículos 1 y 5 . Congreso de la República.

Ley 181 de 1995, artículo 16. Congreso de la República.

Decreto 2225 de 1985. Congreso de la República.

Ley 934 de 2004. Por la cual se oficializa la Política de Desarrollo Nacional de la Educación Física. Congreso de la República.

Decreto reglamentario 1227 DE 1995, artículos 1 y 2. Congreso de la República. 